\title{
Inflammatory Cell Infiltration of Adrenals in COVID-19
}

Authors

Vsevolod A. Zinserling1, 2, Natalya Yu. Semenova1, 2, Alexander G. Markov³, Oksana V. Rybalchenko4 ${ }^{4}$ Jun Wang ${ }^{5}$, Roman N. Rodionov6, 7, Stefan R. Bornstein ${ }^{5,8,9}$

Affiliations

1 S. P. Botkin Clinical Infectious Diseases Hospital, St. Petersburg, Russia

2 V. A. Almasov Scientific Research Center, St. Petersburg, Russia

3 Department of General Physiology, St. Petersburg State University, St. Petersburg, Russia

4 Faculty of Medicine, Department of General Physiology, St. Petersburg State University, St. Petersburg, Russia

5 Department of Internal Medicine III, University Hospital Carl Gustav Carus, Technische Universität Dresden, Dresden, Germany

6 Division of Angiology, Department of Internal Medicine III, University Hospital Carl Gustav Carus, Technische Universität Dresden, Dresden, Germany

7 College of Medicine and Public Health, Flinders University and Flinders Medical Centre, Adelaide, Australia

8 Department of Diabetes, School of Life Course Science and Medicine, King's College London, London, UK

9 Clinic for Endocrinology, Diabetology and Clinical Nutrition, University Hospital, Zurich, Switzerland

Key words

ACE2, adrenal, infiltrations, COVID-19

received $\quad 05.05 .2020$

accepted $\quad 01.06 .2020$

Bibliography

DOI https://doi.org/10.1055/a-1191-8094

Published online: 7.7.2020

Horm Metab Res 2020; 52: 639-641

(c) Georg Thieme Verlag KG Stuttgart · New York

ISSN 0018-5043
Correspondence

Stefan R. Bornstein

Department of Medicine University Hospital Carl Gustav

Carus, University of Dresden

Fetscherstrasse 74

01307 Dresden

Germany

Tel.: + 493514585955 , Fax: + 493514586398

stefan.bornstein@uniklinikum-dresden.de

\section{ABSTRACT}

Severe acute respiratory syndrome coronavirus 2 (SARS-CoV-2) was originated in November-December 2019 in Wuhan, China, and has rapidly spread around the world causing severe health and socioeconomical damage to the entire civilization. The key feature of coronavirus disease 2019 (COVID-19), caused by SARS-CoV-2, is upper respiratory tract infection, which may be complicated by bilateral pneumonia. Angiotensin converting enzyme 2 (ACE2) has been identified as a key host factor, required for virus entry into cells. Interestingly, ACE2 is expressed not only in the respiratory system, but also in the other organs and systems including adrenal glands. Here we provide the first description of the pathomorphological changes in adrenal glands in patients with severe COVID-19 characterized by perivascular infiltration of CD3 + and CD8 + T-lymphocytes. Due to the central role of the adrenals in the stress response of the organism, this finding is of potential clinical relevance, because infection with the SARS-CoV-2 virus might critically impair adrenal function under pathophysiological conditions.

\section{Introduction}

The unprecedented development of the pandemic coronavirus disease 2019 (COVID-19) has made a better understanding of the interaction between the severe acute respiratory syndrome virus coronavirus 2 (SARS-CoV-2) and the host organism a top priority. SARS-CoV-2 is a positive-sense single-stranded RNA virus, which uses the angiotensin converting enzyme 2 (ACE2), a transmembrane protein as a host receptor for cellular entry. The virus has been thought to primarily target the respiratory system, causing the wide range of clinical manifestations from upper respiratory tract infections to bilateral pneumonias with severe acute respiratory distress syndrome (ARDS). Recent reports have demonstrated that SARS-CoV-2 can directly infect other ACE2 expressing cell types, such as renal tubular epithelial cells, podocytes, and endothelial cells [1,2]. Furthermore, a direct metabolic and endocrine link to coronavirus infection has been reported [3,4].

Previous studies have reported strong expression of ACE2 in endocrine organs including the adrenal glands [5]. Although it was 
shown that one of the previous coronaviruses SARS-CoV, which was responsible for the SARS 2002-2004 outbreak, could directly injure adrenal glands [6], morphological changes of the adrenal glands in the course of COVID-19 infection have not been systematically investigated so far.

This is of potential clinical relevance, because the adrenal glands play a central role in the physiology of the stress response of the organism in health and disease. The latter is highly important in the course of severe COVID-19 infection, for example in the regulation of the immune response, and in particular in the control of cytokine release in septic shock, regulation of fluid homeostasis, control of blood pressure, and production of sex hormones, which might be responsible for gender-associated difference in disease progress and survival as observed in COVID-19 patients [7].

Particularly, an adequate adrenal hormone response is crucial for survival of severe infection and septic shock [8]. The adrenals and glucocorticoid hormone signaling pathways are targeted by various bacterial and viral agents thus employing a primary immunoinvasive strategy to blunt the organism's life-saving glucocorticoid stress response $[9,10]$.

Taking into consideration the high vulnerability of adrenal tissue and its crucial role in critical illness a thorough examination of adrenal morphology in patients severely affected by COVID-19 should be of great relevance. Therefore, in the current study, we provide the first systematic morphological report of the pathoimmunological changes in the adrenal glands in a series of autopsies of COVID-19 patients.

\section{Materials and Methods}

Autopsies of 10 patients deceased from COVID-19 were performed in the department of pathology at S. P. Botkin Infectious Hospital according to the national rules, regulating such procedure.

\section{Tissue samples and immunohistochemistry}

In addition to other organs, special attention was paid to the morphology of the adrenal glands. The adrenals of 10 patients were collected and processed for histological and immunohistochemical analysis according to standard protocols. During the autopsy, the macroscopical changes of the adrenals seemed to be unremarkable. Paraffin slices were stained by hematoxylin-eosine. For immune histochemistry we used commercial antibodies against CD3,
CD8, and CD20 (IS503, IS623, and IS604, Dako, Denmark) using standard positive and negative controls in parallel. HIER antigen retrieval was performed using EnVision FLEX Target Retrieval Solution (K8010, Dako, Denmark) according to the protocols of manufacturer. Images were taken using Nikon Eclipse Ni-U microscope (Nikon, Japan).

\section{Results and Discussion}

In the present work, we demonstrate that the adrenal glands can be seriously damaged with distinct structural changes in the course of COVID-19. This involves both: direct lesions due to generalization of the infection and involvement of CD8 + T-lymphocytes.

In a detailed postmortem study, we succeeded to describe two types of adrenal lesions. Most striking was a mononuclear infiltration, which could be detected in the different layers of the organ ( $\triangleright$ Fig 1a) and in surrounding tissue as well. Immunohistochemistry allowed us to determine the exact cell types of the infiltration: CD3 + and CD8 + ( $\mathbf{F i g} \mathbf{1 b})$. We observed changes of the adrenal cortex with formations of small proliferations of cells with enlarged light nuclei. Such changes are similar to those observed in the lungs, which we consider to be caused by a direct action of SARSCoV-2 (> Fig 1c).

Further studies will be required to prove the presence of SARSCoV-2 in adrenal tissue and to define the mechanisms of adrenal degeneration and the potential loss of function. Autopsy studies of patients who passed away from SARS exhibited degeneration and necrosis of adrenal cortical cells. Indeed the SARS virus had been identified in adrenal cells suggesting a direct cytopathic effect of the virus in adrenal tissue. ACE2, which serves as the receptor of entry for SARS-CoV-2 is highly expressed [6] in human adrenals, thereby potentially further exposing adrenal tissue to virus-mediated destruction. Moreover, the SARS virus contains several permutations of amino acid sequences with homology to the antigenic relevant residues of ACTH. This molecular mimicry is of potential pathophysiological relevance, since ACTH is the key hormone regulating adrenal glucocorticoid release and it has been discussed that autoantibodies binding ACTH could abrogate the adrenal stress response. Further, potential cross-reactivity of antibodies may explain local infiltrations with immunocompetent cells in the pituitary and adrenal. In summary, these aspects imply that patients with COVID-19 may be susceptible for critical illness-re-
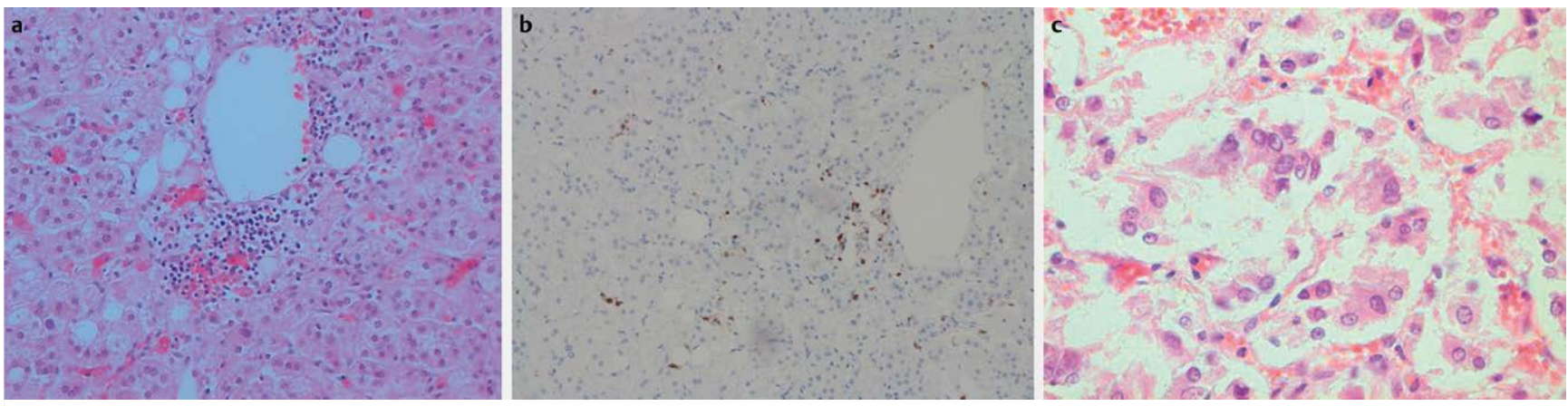

Fig. 1 a Perivascular mononuclear infiltration in adrenal of the deceased with COVID-19. H.-E., Magnification $400 \times$. b Diffuse infiltration of adrenal tissue by CD3 + lymphocytes. IHC, Magnification 100×. c Small cell proliferations in adrenals cortex. H.-E., Magnification 400× . 
lated corticosteroid insufficiency (CIRCI) due to cellular destruction of adrenal tissue by direct virus infection and further due to secondary inflammatory and autoimmune processes located in the pituitary and the adrenals.

Finally, the adrenal gland is one of the most highly vascularized organs of the human body with almost every adrenal cell in very close location to endothelial cells. Therefore, the well-described endotheliitis in COVID-19 disease may further increase the vulnerability of adrenal tissue. This may result not only in adrenal degeneration but possibly also in adrenal hemorrhage and a WaterhouseFriderichsen syndrome. Although we have not yet observed this in our patients it cannot be ruled out in a subset of patients. It may explain some of the severe symptoms of a Kawasaki-like syndrome with severe and acute hypotensive shock responding to glucocorticoid treatment recently reported in some children. Adrenal lesions due to influenza and other viruses have been described previously $[9,10]$.

In conclusion, our data demonstrate an impairment of the adrenals in COVID-19. Hypocortisolism has been observed in SARS survivors 3 months after their recovery [11]. Therefore, adrenal insufficiency should be considered in patients with suggestive symptoms and signs even months after infection with SARS-CoV-2. Further, in COVID-19 patients receiving antiviral medications and/or steroids long-term consequences on HPA axis dysfunction need to be considered [12] to avoid an adrenal crisis. Finally, dysregulation of the HPA axis may result in an aggravation of other chronic disorders including cardiometabolic and mental disease [13].

\section{Acknowledgment}

This work was supported by Transcampus and the Deutsche Forschungsgemeinschaft (GRK 2251/1, TRR 205/1).

\section{Conflict of Interest}

The authors declare that they have no conflict of interest.

\section{References}

[1] Su H, Yang M, Wan C et al. Renal histopathological analysis of 26 postmortem findings of patients with COVID-19 in China. Kidney Int 2020; https://10.1016/j.kint.2020.04.003

[2] Varga Z, Flammer AJ, Steiger P et al. Endothelial cell infection and endotheliitis in COVID-19. Lancet 2020; 395: 1417-1418

[3] Bornstein SR, Rubino F, Khunti K et al. Practical recommendations for the management of diabetes in patients with COVID-19. Lancet Diabetes Endocrinol 2020; DOI: 10.1016/S2213-8587(20)30152-2

[4] Bornstein SR, Dalan R, Hopkins D et al. Endocrine and metabolic link to coronavirus infection. Nat Rev Endocrinol 2020; 16: 297-298

[5] Li MY, Li L, Zhang Y et al. Expression of the SARS-CoV-2 cell receptor gene ACE2 in a wide variety of human tissues. Infect Dis Poverty 2020; 9: 45

[6] Ding Y, He L, Zhang Q et al. Organ distribution of severe acute respiratory syndrome (SARS) associated coronavirus (SARS-CoV) in SARS patients: Implications for pathogenesis and virus transmission pathways. J Pathol 2004; 203: 622-630

[7] Wenham C, Smith J, Morgan R et al. COVID-19: The gendered impacts of the outbreak. Lancet 2020; 395: 846-848

[8] Bornstein SR. Predisposing factors for adrenal insufficiency. N Engl ] Med 2009; 360: 2328-2339

[9] Zinserling AV, Aksenov OA, Melnikova VF et al. Extrapulmonary lesions in influenza. Tohoku J Exp Med 1983; 140: 259-272

[10] Zinserling AV, Zinserling VA. Modern Infections: Pathologic Anatomy and Issues of Pathogenesis. Manual. $2^{\text {nd }}$ ed. Spb, Sotis; 2002: 352

[11] Leow MK, Kwek DS, Ng AW et al. Hypocortisolism in survivors of severe acute respiratory syndrome (SARS). Clin Endocrinol (Oxf) 2005; 63: 197-202

[12] Bornstein SR, Bornstein TD, Andoniadou CL. Novel medications inducing adrenal insufficiency. Nat Rev Endocrinol 2019; 15: 561-562

[13] Steenblock C, Todorov V, Kanczkowski W et al. Severe acute respiratory syndrome coronavirus 2 (SARS-CoV-2) and the neuroendocrine stress axis. Mol Psychiatr 2020; https://doi.org/10.1038/ s41380-020-0758-9 